\title{
The role of the macrophage in sentinel responses in intestinal immunity
}

\author{
Shehzad Z. Sheikh and Scott E. Plevy \\ Department of Medicine, Microbiology and Immunology, University of North Carolina at Chapel \\ Hill School of Medicine, Chapel Hill, North Carolina, USA
}

\begin{abstract}
Purpose of review-The purpose of this review is to highlight macrophages as central mediators of intestinal immune homeostasis and inflammation.

Recent findings-We review recent developments elucidating distinct phenotypic adaptations in intestinal macrophages that determine their functional role in a microbe-rich environment. The involvement of intestinal macrophages in the pathogenesis of inflammatory bowel disease is also discussed.
\end{abstract}

Summary-Intestinal macrophages represent the largest pool of tissue macrophages in the human body and a critical interface with the enteric microbiota. In normal physiology, luminal microbes breach the intestinal epithelial barrier and gain access to the lamina propria. Bacteria are efficiently phagocytosed by macrophages strategically located underneath the epithelium. The importance of functional adaptations of macrophages to perform their role in this unique environment is best illustrated by failure of these mechanisms during the development of chronic inflammatory bowel diseases. Compared with monocytes or macrophages from any other organ, intestinal macrophages express different phenotypic markers, efficiently eradicate intracellular bacteria, but do not mount potent inflammatory responses. Converging human genetic and functional findings suggest that dysregulation of macrophage-specific immune responses against an otherwise harmless enteric microbiota are key factors in the pathogenesis of inflammatory bowel disease.

\section{Keywords}

inflammatory bowel disease; innate immunity; macrophage

\section{Introduction}

The innate immune system contributes to the functional integrity of the intestinal mucosa in health and disease through monitoring of luminal contents, particularly the enteric microbiota. Key participants in intestinal innate immune defenses are macrophages. Innate responses are rapid and directed toward conserved patterns of carbohydrate and lipid structures on infectious agents [pathogen-associated molecular patterns, or (PAMPs)] recognized by germ line-encoded pattern recognition receptors, toll-like receptors (TLRs) and nod-like receptors (NLRs) [1]. Engagement of these receptors stimulates signaling cascades that include nuclear factor- $\kappa \mathrm{B}$, serine/threonine protein kinase (AKT)/

(C) 2010 Wolters Kluwer Health | Lippincott Williams \& Wilkins

Correspondence to Dr Scott E. Plevy, MD, Department of Medicine, Microbiology and Immunology, University of North Carolina at Chapel Hill School of Medicine, CB 7032, 7341c MBRB, 103 Mason, Farm Road, Chapel Hill, NC 27599, USA, Tel: +1 919 966 4405; fax: +1 919843 2585; scott_plevy@med.unc.edu. 
phosphoinositide-3'-kinase, and mitogen-activated protein kinase pathways [2]. Mutations in TLRs and NLRs have been associated with inflammatory bowel disease (IBD), suggesting that this innate immune detection system is key for regulating mucosal homeostasis [3].

The intestinal innate immune system is in a default state of hyporesponsiveness. Intestinal macrophages show attenuated proliferation and chemotactic activity in response to microbial ligands and cytokines/chemokines despite possessing the molecular mechanisms to elaborate strong phagocytic and bactericidal responses [4]. However, in IBD, following an inflammatory signal, circulating monocytes migrate to the intestinal mucosa and, unlike resident macrophages, are capable of a rapid response to luminal microbial triggers [5].

\section{Current views on macrophage development and biology}

A common myeloid progenitor cell gives rise to monocytes, which are released from the bone marrow into the bloodstream. These peripheral blood monocytes migrate to tissues under the steady state or in response to inflammation. Innate and adaptive signals can then influence macrophage physiology, and these alterations allow macrophages to participate in homeostatic processes, such as tissue remodeling, wound healing, and host defense. Macrophages also have remarkable plasticity that allows them to efficiently respond to environmental signals and change their functional characteristics.

Macrophages have been somewhat simplistically classified as M1 [high interleukin (IL)-12 and low IL-10 producing] macrophages and M2 macrophages (low IL-12 and high IL-10 producing). M1 or classically activated macrophages develop during cell-mediated immune responses requiring both interferon (IFN)- $\gamma$ and tumor necrosis factor (TNF). Classically activated macrophages form an integral component of host defense through production of inflammatory cytokines such as IL-1, IL-6, IL-12, and IL-23 [6]. Phenotypically, M1 macrophages are identified by the expression of inducible nitric oxide synthase, C-C motif chemokine 15 (CCL15) and 20 (CCL20), $\gamma$-interferon-induced monokine 9 (CXCL9) and 10 (CXCL10) [6]. M1 macrophages are essential for eradication of intracellular microorganisms $\left[7^{\circ}\right]$. However, they are also mediators of pathology that occurs during chronic inflammatory disorders, including IBD.

M2 or alternatively activated macrophages represent a wide array of macrophage phenotypes [6]. This spectrum includes macrophages with wound healing and regulatory properties [6]. In response to injury, early increases in IL-4 and/or IL-13 rapidly convert resident macrophages into a population of cells programmed to promote wound healing through production of extracellular matrix [8]. Regulatory macrophages that arise during innate or adaptive immune responses, under the influence of IL-10 and transforming growth factor (TGF)- $\beta$, dampen the immune response and limit inflammation [6]. Production of IL-10, TGF- $\beta$, PGE $_{2}$, and the ability to suppress IL-12 production are hallmarks of regulatory macrophages [9]. Phenotypically, M2 macrophages are identified by the expression of arginase 1, chitinase-like protein that can bind to extracellular matrix, and found in inflammatory zone 1 [10].

\section{Microbial recognition and eradication by macrophages}

Macrophages are essential for the phagocytosis and clearance of enteric bacteria that breach the intestinal epithelial barrier [11]. Autophagy and phagolysosomal function have emerged as central components of the macrophage machinery to eradicate intracellular bacteria [12]. Indeed, the importance of microbicidal pathways in the pathogenesis of IBD is highlighted by the discovery that synonymous single nucleotide polymorphisms in the autophagocytic genes ATG16LI and IRGM and the phagosomal gene NCF4 are associated with enhanced 
risk for IBD [1,12]. Moreover, the Crohn's disease susceptibility gene $\mathrm{N}$ has recently been linked to autophagy $\left[13^{\circ}\right]$. Autophagy, directly translated as 'self-eating', is a process whereby cells digest their own organelles or cytoplasm, allowing survival during periods of nutrient deprivation. Importantly, autophagy is required to protect against bacteria that invade cells, and recent functional analyses suggest that Crohn's disease-associated autophagy mutations may impair the ability of cells to eradicate intracellular bacteria $[14,15]$. Signaling through NLRs and TLRs can lead to autophagosome formation $\left[13^{\circ}, 16\right]$. During autophagy, cytoplasmic material including organelles, protein aggregates, or bacteria is sequestered into double membrane-bound autophagosomes that subsequently fuse with endosomes and lysosomes to form autolysosomes, where lysosomal degradation occurs. Autophagy also has a major role in antigen presentation, with fusion of autophagosomes with multivesicular major histocompatibility complex (MHC) class II loading compartments in macrophages [17].

A fundamental question in mucosal immunity is how mucosal innate host defense distinguishes pathogenic from resident microbes. It is not known how pattern recognition receptors such as NLRs and TLRs differentiate conserved molecular patterns on pathogenic and commensal bacteria, nor is it known how this recognition process may be altered in IBD.

\section{Distinct properties of resident intestinal macrophages}

The local intestinal microenvironment substantially influences the differentiation of macrophages. Intestinal macrophages express high levels of MHC class II and the myeloid marker aminopeptidase $\mathrm{N}$ (CD13), similarly to blood monocytes (Table 1) [18-26]. However, in contrast to their progenitor cells, resident monocytes, intestinal macrophages do not function as professional antigen-presenting cells (APC) owing to their low cell surface expression of the costimulatory molecules CD40, CD80, and CD86 [20]. They also lack the Fc receptors for immunoglobulin A (CD89) and for immunoglobulin G (CD16, CD32, and CD64) and the complement receptors CR3 (CD11b/CD18) and CR4 (CD11c/CD18). Similarly, most intestinal macrophages lack the integrin $\alpha 2 \beta 1$ (lymphocyte functionassociated antigen-1 and CD11a/CD18) [20,21]. These receptors for the $\mathrm{Fc}$ and complement family members on macrophages mediate cellular activation, secretion of proinflammatory cytokines (TNF, IL-1 $\beta$, IL-6, and IL-12) and drive adaptive immune responses. Intestinal macrophages also lack the triggering receptor expressed on myeloid cells-1 (TREM-1), an efficient amplifier of acute and chronic inflammatory reactions that is expressed on most monocytes and macrophages in secondary lymphoid organs [22]. Engagement of TREM-1 on TREM-1-positive macrophages leads to enhanced TNF, IL-1 $\beta$, and IL-6 secretion. Upregulation of several cell surface molecules (C40, CD86, and CD32) also prolongs survival of TREM-1-positive myeloid cells [22].

The selective absence of these receptors on intestinal macrophages has functional implications in the maintenance of immune homeostasis. Intestinal macrophages under physiological conditions are refractory to the induction of inflammatory cytokines by PAMPs, cytokines (e.g., TNF, IFN- $\gamma$ ), and phagocytosis of necrotic cells [4]. This is in sharp contrast to other tissue macrophages and blood monocytes. Intestinal epithelial cells, subepithelial myofibroblasts, fibroblasts, lamina propria lymphocytes, and intraepithelial lymphocytes also produce soluble factors, particularly TGF- $\beta$ and IL-10, which determine the functional properties of intestinal macrophages [27,28]. However, these adaptations made by intestinal macrophages do not impair phagocytic activity. Intestinal macrophages are highly effective at killing phagocytosed enteric bacteria such as Salmonella typhimurium and Escherichia coli [21]. 


\section{Regulation of intestinal macrophage phenotype and function}

The local intestinal milieu shapes the function and phenotype of intestinal macrophages. Blood monocytes treated with intestinal stromal cell-conditioned media rich in TGF- $\beta$ acquire a tolerized state with reduced expression of innate immune receptors and increased phagocytotic activity, similar to intestinal macrophages. Blocking TGF- $\beta$ reverses these effects [21]. Similarly, IL-10, produced under physiological conditions in the intestinal lamina propria, directly dampens macrophage function and upregulates TGF- $\beta$ production in multiple cell types [29]. Interestingly, monocytes cultured in the presence of TGF- $\beta$ alone or in combination with IL-10 do not acquire an intestinal macrophage phenotype [22]. However, monocytes cultured with multicellular spheroids of intestinal epithelial cells acquire an intestinal macrophage phenotype, characterized by reduced CD14 and reduced lipopolysaccharides-stimulated IL-1 $\beta$ expression [30].

Macrophages also maintain intestinal immune homeostasis through the generation of anergic and/ or regulatory $\mathrm{T}$ cells in the intestine, and fine tuning adaptive immune responses by altering the Th1/Th2/Th17 balance [31]. Retinoic acid directly influences the development and effector functions of intestinal macrophages. A population of $\mathrm{CD} 11 \mathrm{~b}^{+} \mathrm{F} 4 / 80^{+} \mathrm{CD} 11 \mathrm{c}^{-}$ intestinal macrophages that constitutively produce high levels of IL-10 express high levels of Aldhlal and Aldhla2, retinol dehydrogenases that convert retinol into retinoic acid, and induce T-regulatory cells $[32,33]$. These macrophages convert naïve $\mathrm{CD} 4^{+} \mathrm{T}$ cells to Foxp $3^{+}$ $\mathrm{T}$ cells in vitro in the presence of exogenous TGF- $\beta$ via a mechanism dependent on both IL-10 and Aldhlal and Aldhla2 [33]. Importantly, not all intestinal APCs induce Tregulatory cells. $\mathrm{CD} 11 \mathrm{c}^{+} \mathrm{CD} 11 \mathrm{~b}^{+}$subset of dendritic cells in the lamina propria induces robust Th17 responses [33]. These studies emphasize the complexity of the intestinal APC network that differentially regulates mucosal immune responses.

\section{Lessons learned from murine models of experimental colitis}

Murine models of experimental colitis have demonstrated the importance of macrophage regulation for maintaining local tissue homeostasis. Selective disruption of Stat3 leads to impaired IL-10 signaling in macrophages and consequently leads to the development of colitis [34]. Similarly, IL-10 $10^{-1-}$ mice spontaneously develop colitis as a consequence of the preferential macrophage differentiation into proinflammatory subsets that produce large amounts of IL-12 and IL-23 [35]. Depletion of macrophages in IL-10 $0^{-/-}$mice prevents the development of colitis [35].

Of the inflammatory genes induced in macrophages, IL-23 has been strongly implicated in the pathogenesis of murine and human IBD [3]. IL-23 promotes a distinct CD4 ${ }^{+} \mathrm{T}$-cell phenotype characterized by the production of the cytokine IL-17, denoted Th17 cells. IL-23 enhances Th17 function and survival by acting on differentiated Th17 cells that express the IL-23 receptor. Indeed, IFN- $\gamma$, the signature Th1 cytokine induced by IL-12, and IL-10 were both recently shown to inhibit IL-23 in lamina propria macrophages [ $\left.36^{\circ}\right]$. As an illustration of the functional adaptation of intestinal macrophages to their local environment, murine colonic macrophages produce large amounts of IL-10 after encountering the enteric microbiota [35]. In the context of adaptive immune responses, IFN- $\gamma$ has been considered to be a proinflammatory cytokine, driving Th1 responses. However, the enteric microbiota transiently induces IFN- $\gamma$ expression in the colon of wild type germfree mice colonized with the enteric microbiota, which in turn attenuated IL-23 in macrophages, suggesting that local macrophage-specific regulatory check points are vital in maintaining immune homeostasis $\left[36^{\circ}\right]$.

The importance of intestinal macrophages in maintaining adaptive immune homeostasis was recently highlighted in studies showing that IL-10 secreted from resident intestinal 
macrophages acts in a paracrine manner on regulatory $\mathrm{T}$ (Treg) cells to maintain Foxp3 expression [ $\left[7^{\circ}\right]$. These Foxp3 expressing Treg cells have been shown to suppress the activity of Th1 and Th17 cells in inflamed tissues [1].

\section{Intestinal macrophages in human inflammatory bowel disease}

Intestinal inflammation in the human IBDs, Crohn's disease and ulcerative colitis, results from an inappropriately directed inflammatory response to the enteric microbiota in a genetically susceptible host. Genome wide association studies have identified IBD susceptibility genes that affect barrier function and innate and adaptive immune responses in the intestine. Genetic variants that confer Crohn's disease risk highlight the importance of innate immunity, including NOD2, NCX5, IRGM, and ATG16L1 [3].

In patients with active IBD, macrophage numbers are increased in inflamed intestinal mucosa [1]. Many of these macrophages display a different phenotypic and functional profile than under homeostatic conditions. Macrophages in the inflamed intestine express functional $\mathrm{T}$ cell costimulatory molecules such as CD40, CD80, and CD86 [18]. Furthermore, subsets of intestinal macrophages express TLR2, TLR4, CD89, and TREM-1 at the site of intestinal inflammation [19]. Similarly, a high proportion of the macrophages from patients with IBD express CD14 [20,24]. CD14 expressing macrophages (CD14+) infiltrating the mucosa in IBD produce larger amounts of IL-12, IL-23, and TNF compared with intestinal macrophages from healthy controls. These CD14+ macrophages produce IFN- $\gamma$ that further triggers abnormal macrophage differentiation with an IL-23-hyperproducing phenotype [35]. In an inflammatory milieu, the close proximity of subepithelial macrophages to the intestinal microbiota results in an excessive activation of the mucosal immune system [35]. CD14+ intestinal macrophages from Crohn's disease patients respond vigorously to in-vitro microbial stimulation with production of abundant TNF, IL-12, and IL-23, compared with CD14- intestinal macrophages [24]. CD14+ intestinal macrophages also express TREM-1. TREM-1 triggers the synthesis and secretion of inflammatory factors including IL-1 $\beta$, monocyte chemotactic protein-1 (MCP-1), IL-6, and IL-8 [5]. Macrophages are also the main source of TNF during the pathogenesis of IBD. The striking clinical benefit of TNF-targeted monoclonal antibody therapies may be attributed to the celldepleting effects, including the removal of TNF membrane bound expressing macrophages [38].

An impaired ability to eradicate intracellular pathogens by macrophages in IBD may also be secondary to their inability to secrete proinflammatory cytokines in response to bacteria or TLR agonists. Provocatively, it has been speculated that, despite normal levels and stability of cytokine mRNA, intracellular levels of TNF are abnormally low in macrophages from Crohn's disease patients. Coupled with reduced secretion, these findings indicate that accelerated intracellular defects in genes encoding proteins involved in vesicle trafficking may result in an abnormal proportion of cytokines being routed to lysosomes and degraded rather than being released through the normal secretory pathway $\left[7^{\circ}\right]$.

The concept that macrophages from patients with IBD display distinct functional characteristics was further supported by studies demonstrating mutations in genes encoding the IL-10 receptor (IL-10R) subunit proteins IILIORA and ILIORB in patients with earlyonset enterocolitis [39*0. Increased secretion of TNF, macrophage inflammatory protein-1 (MIP1) $\alpha$, MIP1 $\beta$, MCP-1 and chemokine (C-C motif) ligand 5 (RANTES), and failure to downregulate inflammatory cytokine expression by IL-10 in peripheral blood monocytes from patients who were deficient in IL-10R subunit proteins, again demonstrate altered macrophage responses that may underlie IBD pathogenesis. Interestingly, in this study, 
remission was achieved in one patient following allogeneic bone marrow transplantation, correcting the IL-10 signaling defect in macrophages [39*0].

\section{Conclusion}

Until recently, research on the pathogenesis of IBD has focused on effector mechanisms of the adaptive immune system. The relevance of macrophages in IBD is highlighted by their functions as gatekeepers, driving (not bridging) adaptive immune responses to either tolerance or initiation of inflammation. Detailed mechanisms of how intestinal macrophages functionally differentiate and participate in the pathogenesis of IBD may suggest specific strategies that target induction of macrophage specific regulatory responses and prevent chronic inflammation.

Vital to the development of such therapies is an intricate knowledge about the monocyte/ macrophage lineage relationships, differential homing capacities, and the effects of the local cytokine milieu on macrophage function and phenotype, and the plasticity of intestinal macrophage subsets.

\section{Acknowledgments}

S.E.P. is supported by a grant from the National Institutes of Health (NIH)(RO1 DK54452). S.Z.S. is supported by the NIH Gastroenterology Research Training Grant (T32 DK007737) and National Research Service Award (F32 DK083186).

\section{References and recommended reading}

Papers of particular interest, published within the annual period of review, have been highlighted as:

- of special interest

•- of outstanding interest

Additional references related to this topic can also be found in the Current World Literature section in this issue (pp. 659-660).

1. Xavier RJ, Podolsky DK. Unravelling the pathogenesis of inflammatory bowel disease. Nature. 2007; 448:427-434. [PubMed: 17653185]

2. Akira S, Takeda K. Toll-like receptor signalling. Nat Rev Immunol. 2004; 4:499-511. [PubMed: 15229469]

3. Cho JH. The genetics and immunopathogenesis of inflammatory bowel disease. Nat Rev Immunol. 2008; 8:458-466. [PubMed: 18500230]

4. Smith PD, Ochsenbauer-Jambor C, Smythies LE. Intestinal macrophages: unique effector cells of the innate immune system. Immunol Rev. 2005; 206:149-159. [PubMed: 16048547]

5. Schenk M, Bouchon A, Seibold F, et al. TREM-1-expressing intestinal macrophages crucially amplify chronic inflammation in experimental colitis and inflammatory bowel diseases. J Clin Invest. 2007; 117:3097-3106. [PubMed: 17853946]

6. Mosser DM, Edwards JP. Exploring the full spectrum of macrophage activation. Nat Rev Immunol. 2008; 8:958-969. [PubMed: 19029990]

7•. Smith AM, Rahman FZ, Hayee B, et al. Disordered macrophage cytokine secretion underlies impaired acute inflammation and bacterial clearance in Crohn's disease. J Exp Med. 2009; 206:1883-1897. This study implicates defects in vesicle trafficking in macrophages from Crohn's disease patients through abnormal routing of a major proportion of cytokines to lysosomes and degradation rather than being released through the normal secretory pathway. [PubMed: 19652016] 
8. Kreider T, Anthony RM, Urban JF Jr, et al. Alternatively activated macrophages in helminth infections. Curr Opin Immunol. 2007; 19:448-453. [PubMed: 17702561]

9. Gerber JS, Mosser DM. Reversing lipopolysaccharide toxicity by ligating the macrophage Fc gamma receptors. J Immunol. 2001; 166:6861-6868. [PubMed: 11359846]

10. Raes G, De Baetselier P, Noel W, et al. Differential expression of FIZZ1 and Ym1 in alternatively versus classically activated macrophages. J Leukoc Biol. 2002; 71:597-602. [PubMed: 11927645]

11. Hisamatsu T, Ogata H, Hibi T. Innate immunity in inflammatory bowel disease: state of the art. Curr Opin Gastroenterol. 2008; 24:448-454. [PubMed: 18622158]

12. Rioux JD, Xavier RJ, Taylor KD, et al. Genome-wide association study identifies new susceptibility loci for Crohn disease and implicates autophagy in disease pathogenesis. Nat Genet. 2007; 39:596-604. [PubMed: 17435756]

13. Cooney R, Baker J, Brain O, et al. NOD2 stimulation induces autophagy in dendritic cells influencing bacterial handling and antigen presentation. Nat Med. 2010; 16:90-97. This study is the first to link two Crohn's disease-associated susceptibility genes, NOD2 and ATG16L1, in a single functional pathway and reveals defects in autophagy leading to bacterial persistence in dendritic cells via impaired lysosomal destruction and immune mediated clearance. [PubMed: 19966812]

14. Kuballa P, Huett A, Rioux JD, et al. Impaired autophagy of an intracellular pathogen induced by a Crohn's disease associated ATG16L1 variant. PLoS ONE. 2008; 3:e3391. [PubMed: 18852889]

15. Xavier RJ, Huett A, Rioux JD. Autophagy as an important process in gut homeostasis and Crohn's disease pathogenesis. Gut. 2008; 57:717-720. [PubMed: 18272545]

16. Blander JM, Medzhitov R. Toll-dependent selection of microbial antigens for presentation by dendritic cells. Nature. 2006; 440:808-812. [PubMed: 16489357]

17. Levine B, Kroemer G. Autophagy in the pathogenesis of disease. Cell. 2008; 132:27-42. [PubMed: 18191218]

18. Rugtveit J, Bakka A, Brandtzaeg P. Differential distribution of B7.1 (CD80) and B7.2 (CD86) costimulatory molecules on mucosal macrophage subsets in human inflammatory bowel disease (IBD). Clin Exp Immunol. 1997; 110:104-113. [PubMed: 9353156]

19. Hausmann M, Kiessling S, Mestermann S, et al. Toll-like receptors 2 and 4 are up-regulated during intestinal inflammation. Gastroenterology. 2002; 122:1987-2000. [PubMed: 12055604]

20. Smith PD, Smythies LE, Mosteller-Barnum M, et al. Intestinal macrophages lack CD14 and CD89 and consequently are down-regulated for LPS- and IgA-mediated activities. J Immunol. 2001; 167:2651-2656. [PubMed: 11509607]

21. Smythies LE, Sellers M, Clements RH, et al. Human intestinal macrophages display profound inflammatory anergy despite avid phagocytic and bacteriocidal activity. J Clin Invest. 2005; 115:66-75. [PubMed: 15630445]

22. Schenk M, Bouchon A, Birrer S, et al. Macrophages expressing triggering receptor expressed on myeloid cells-1 are underrepresented in the human intestine. J Immunol. 2005; 174:517-524. [PubMed: 15611278]

23. Smith PD, Janoff EN, Mosteller-Barnum M, et al. Isolation and purification of CD14-negative mucosal macrophages from normal human small intestine. J Immunol Methods. 1997; 202:1-11. [PubMed: 9075766]

24. Kamada N, Hisamatsu T, Okamoto S, et al. Unique CD14 intestinal macrophages contribute to the pathogenesis of Crohn disease via IL-23/IFN-gamma axis. J Clin Invest. 2008; 118:2269-2280. [PubMed: 18497880]

25. Meng G, Sellers MT, Mosteller-Barnum M, et al. Lamina propria lymphocytes, not macrophages, express CCR5 and CXCR4 and are the likely target cell for human immunodeficiency virus type 1 in the intestinal mucosa. J Infect Dis. 2000; 182:785-791. [PubMed: 10950772]

26. Rogler G, Hausmann M, Vogl D, et al. Isolation and phenotypic characterization of colonic macrophages. Clin Exp Immunol. 1998; 112:205-215. [PubMed: 9649182]

27. Khoo UY, Proctor IE, Macpherson AJ. CD4+ T cell down-regulation in human intestinal mucosa: evidence for intestinal tolerance to luminal bacterial antigens. J Immunol. 1997; 158:3626-3634. [PubMed: 9103424] 
28. Maloy KJ, Powrie F. Regulatory T cells in the control of immune pathology. Nat Immunol. 2001; 2:816-822. [PubMed: 11526392]

29. Moore KW, O'Garra A, de Waal Malefyt R, et al. Interleukin-10. Annu Rev Immunol. 1993; 11:165-190. [PubMed: 8386517]

30. Rogler G, Gelbmann CM, Vogl D, et al. Differential activation of cytokine secretion in primary human colonic fibroblast/myofibroblast cultures. Scand J Gastroenterol. 2001; 36:389-398. [PubMed: 11336164]

31. Manicassamy S, Pulendran B. Retinoic acid-dependent regulation of immune responses by dendritic cells and macrophages. Semin Immunol. 2009; 21:22-27. [PubMed: 18778953]

32. Coombes JL, Siddiqui KR, Arancibia-Carcamo CV, et al. A functionally specialized population of mucosal CD103+ DCs induces Foxp3+ regulatory T cells via a TGF-beta and retinoic aciddependent mechanism. J Exp Med. 2007; 204:1757-1764. [PubMed: 17620361]

33. Denning TL, Wang YC, Patel SR, et al. Lamina propria macrophages and dendritic cells differentially induce regulatory and interleukin 17-producing T cell responses. Nat Immunol. 2007; 8:1086-1094. [PubMed: 17873879]

34. Takeda K, Clausen BE, Kaisho T, et al. Enhanced Th1 activity and development of chronic enterocolitis in mice devoid of Stat3 in macrophages and neutrophils. Immunity. 1999; 10:39-49. [PubMed: 10023769]

35. Kamada N, Hisamatsu T, Okamoto S, et al. Abnormally differentiated subsets of intestinal macrophage play a key role in Th1-dominant chronic colitis through excess production of IL-12 and IL-23 in response to bacteria. J Immunol. 2005; 175:6900-6908. [PubMed: 16272349]

36•. Sheikh SZ, Matsuoka K, Kobayashi T, et al. Cutting edge: IFN-gamma is a negative regulator of IL-23 in murine macrophages and experimental colitis. J Immunol. 2010; 184:4069-4073. This study characterizes regulation of mucosal IL-23 by the enteric microbiota and elucidates a protective role for IFN-g in colitis through attenuation of IL-23 expression in lamina propria macrophages. [PubMed: 20228197]

37•. Murai M, Turovskaya O, Kim G, et al. Interleukin 10 acts on regulatory T cells to maintain expression of the transcription factor Foxp3 and suppressive function in mice with colitis. Nat Immunol. 2009; 10:1178-1184. Results from this study identify lamina propria macrophages as the major source of IL-10 in the gut. They also demonstrate that IL-10 released from macrophages acts in a paracrine manner on T(reg) cells to maintain Foxp3 expression. [PubMed: 19783988]

38. van Deventer SJ. Transmembrane TNF-alpha, induction of apoptosis, and the efficacy of TNFtargeting therapies in Crohn's disease. Gastroenterology. 2001; 121:1242-1246. [PubMed: 11677219]

39••. Glocker EO, Kotlarz D, Boztug K, et al. Inflammatory bowel disease and mutations affecting the interleukin-10 receptor. N Engl J Med. 2009; 361:2033-2045. This study is the first to demonstrate that patients with early-onset enterocolitis have mutations in genes encoding the IL10R subunit proteins that result in a hyperinflammatory immune response in the intestine. [PubMed: 19890111] 
Table 1

Distinct phenotypic and functional properties of intestinal macrophages and blood monocytes

\begin{tabular}{lcc}
\hline Phenotype & Intestinal macrophages & Blood monocytes \\
\hline CD14, CD25 & - & + \\
TREM-1, CD69 & - & + \\
CD89, CD16 & - & + \\
CD80, CD86, CD40 & - & + \\
CCR5, CXCR4 & - & + \\
TGF- $\beta$ R1, TGF- $\beta$ RII & + & + \\
CD25, CD123 & - & + \\
HLA-DR, CD33, CD13 & + & + \\
Function & - & + \\
Phagocytosis & + & + \\
Killing & + & + \\
Antigen presentation & - & + \\
Chemotaxis & - & + \\
Costimulation & - & + \\
Cytokine production & - & + \\
\hline
\end{tabular}

This is a summary of functional and phenotypic differences between intestinal macrophages and blood monocytes. Data was collected from references [18-26]. HLA-DR, human leukocyte antigen-DR; TGF, transforming growth factor; TREM-1, triggering receptor expressed on myeloid cells-1. 